\title{
HIGH SPATIAL RESOLUTION NEAR-IR TIP/TILT IMAGING OF "WARM" ULTRALUMINOUS INFRARED GALAXIES
}

\author{
J.A. SURACE AND D.B. SANDERS \\ Institute for Astronomy, University of Hawaii \\ 2680 Woodlawn Dr., Honolulu, HI 96822
}

We present results from high spatial resolution (FWHM $\approx 0.3-0.5$ ") nearIR $(1.6$ and $2.1 \mu \mathrm{m})$ imaging of a complete sample of ultraluminous infrared galaxies (ULIGs) chosen to have "warm" mid-IR colors $\left(f_{25} / f_{60}>0.2\right)$ characteristic of AGN. In conjunction with our WFPC2 imaging program (Surace et al. 1998), we have found that nearly all of these systems are advanced mergers with complex nuclear morphologies. The extended underlying galaxies are detected in each system at $\mathrm{H}$ and $\mathrm{K}^{\prime}$, and are found to have luminosities of a few $L^{*}$, similar to quasars (McLeod \& Rieke 1994). Many of the circumnuclear star-forming knots seen at optical wavelengths have been detected. Based on model SEDs, their bolometric luminosities appear similar to those of the extended nuclear starbursts seen in other, less-luminous interacting systems (i.e. NGC 4038/9). Each ULIG is increasingly dominated at long wavelengths by a compact source which we identify as a putative active nucleus. The optical/near-IR colors of these putative nuclei are more extreme than the most infrared-active starburst galaxies, yet are identical to "far-IR loud" quasars which are in turn similar to optical quasars with significant hot $(800 \mathrm{~K})$ dust emission. Half of the ULIGs have dereddened nuclear near-IR luminosities comparable to those of QSOs, while the others resemble Seyferts; this may be an effect of patchy extinction and scattering. Similarities between the putative ULIG nuclei and QSO nuclei, the underlying host galaxies, and the apparent young age of the ULIGs (as evidenced by their compact star-forming knots) support the evolution of "warm" ULIGs into optical QSOs.

\section{References}

Surace, J.A., Sanders, D.B., Vacca, W.D., Veilleux, S., Mazzarella, J.M. 1998, ApJ, 492, 116

McLeod, K.K. \& Rieke, G.H. 1994, ApJ, 431, 137 